\title{
Analysis of Gumbel Model for Software Reliability Using Bayesian Paradigm
}

\author{
Raj Kumar \\ National Institute of Electronics and \\ Information Technology, \\ Gorakhpur, U.P., India.
}

\author{
Ashwini Kumar Srivastava* \\ Department of Computer Application, \\ Shivharsh Kisan P.G. College, Basti, \\ U.P., India. \\ * Corresponding Author
}

\author{
Vijay Kumar \\ Department of Maths. \& Statistics, \\ D.D.U. Gorakhpur University, \\ Gorakhpur, U.P., India.
}

\begin{abstract}
In this paper, we have illustrated the suitability of Gumbel Model for software reliability data. The model parameters are estimated using likelihood based inferential procedure: classical as well as Bayesian. The quasi NewtonRaphson algorithm is applied to obtain the maximum likelihood estimates and associated probability intervals. The Bayesian estimates of the parameters of Gumbel model are obtained using Markov Chain Monte Carlo(MCMC) simulation method in OpenBUGS(established software for Bayesian analysis using Markov Chain Monte Carlo methods). The $\mathbf{R}$ functions are developed to study the statistical properties, model validation and comparison tools of the model and the output analysis of MCMC samples generated from OpenBUGS. Details of applying MCMC to parameter estimation for the Gumbel model are elaborated and a real software reliability data set is considered to illustrate the methods of inference discussed in this paper.
\end{abstract}

Keywords- Probability density function; Bayes Estimation; Hazard Function; MLE; OpenBUGS; Uniform Priors.

\section{INTRODUCTION}

A frequently occurring problem in reliability analysis is model selection and related issues. In standard applications like regression analysis, model selection may be related to the number of independent variables to include in a final model. In some applications of statistical extreme value analysis, convergence to some standard extreme-value distributions is crucial.

A choice has occasionally to be made between special cases of distributions versus the more general versions. In this chapter, statistical properties of a recently proposed distribution is examined closer and parameter estimation using maximum likelihood as a classical approach by $\mathrm{R}$ functions is performed where comparison is made to Bayesian approach using OpenBUGS.

In reliability theory the Gumbel model is used to model the distribution of the maximum (or the minimum) of a number of samples of various distributions. One of the first scientists to apply the theory was a German mathematician Gumbel[1]. Gumbel focused primarily on applications of extreme value theory to engineering problems. The potential applicability of the Gumbel model to represent the distribution of maxima relates to extreme value theory which indicates that it is likely to be useful if the distribution of the underlying sample data is of the normal or exponential type.

The Gumbel model is a particular case of the generalized extreme value distribution (also known as the Fisher-Tippett distribution)[2]. It is also known as the log-Weibull model and the double exponential model (which is sometimes used to refer to the Laplace model).

It is often incorrectly labelled as Gompertz model [3,4]. The Gumbel model's pdf is skewed to the left, unlike the Weibull model's pdf which is skewed to the right $[5,6]$. The Gumbel model is appropriate for modeling strength, which is sometimes skewed to the left.

\section{MODEL ANALYSIS}

The two-parameter Gumbel model has one location and one scale parameter. The random variable $\mathrm{x}$ follows Gumbel model with the location and scale parameter as $-\infty<\mu<\infty$ and $\sigma>0$ respectively, if it has the following cummulative distribution function(cdf)

$$
\mathrm{F}(\mathrm{x} ; \mu, \sigma)=\exp \{-\exp (-(\mathrm{x}-\mu) / \sigma)\} \quad ; \mathrm{x} \in(-\infty, \infty)
$$

The corresponding probability density function (pdf) is

$$
\mathrm{f}(\mathrm{x} ; \mu, \sigma)=\frac{1}{\sigma} \exp (\mathrm{u})\{\exp (-\exp (\mathrm{u}))\} ; \mathrm{x} \in(-\infty, \infty)
$$

Some of the specific characteristics of the Gumbel model are:

The shape of the Gumbel model is skewed to the left. The pdf of Gumbel model has no shape parameter. This means that the Gumbel pdf has only one shape, which does not change.

The pdf of Gumbel model has location parameter $\mu$ which is equal to the mode but differs from median and mean. This is because the Gumbel model is not symmetrical about its $\mu$.

As $\mu$ decreases, the pdf is shifted to the left. As $\mu$ increases, the pdf is shifted to the right. 


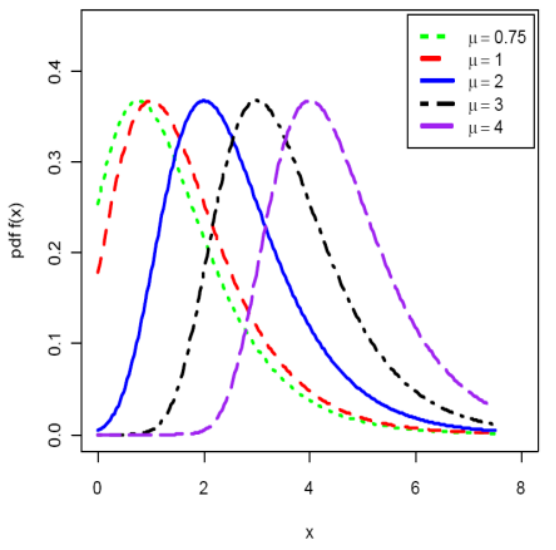

(a)

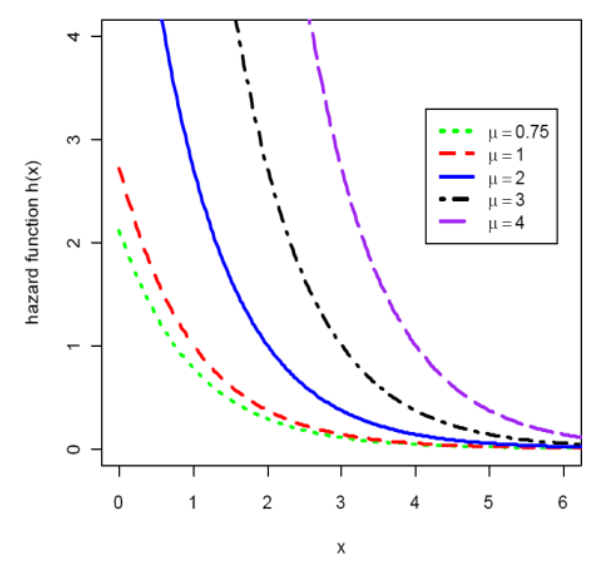

(b)

Figure 1. Plots of the (a) probability density function and (b) hazard function of the Gumbel model for $\sigma=1$ and different values of $\mu$

The hazard function of Gumbel $(\square \mu, \sigma)$ is given by

$$
\mathrm{h}(\mathrm{x})=\frac{1}{\sigma} \exp (-(\mathrm{x}-\mu) / \sigma)
$$

where $\mathrm{x} \in(-\infty, \infty), \mu \in(-\infty, \infty), \sigma>0$

It is clear from the Figure 1 that the density function and hazard function of the Gumbel model can take different shapes. solving

The quantile function of Gumbel model can be obtained by

$$
\mathrm{x}_{\mathrm{p}}=\mu-\sigma[\log (-\log (\mathrm{p}))] \quad ; 0<\mathrm{p}<1 .
$$

The median is

$$
\operatorname{Median}\left(\mathrm{x}_{0.5}\right)=\mu-\sigma \ln \{-\ln (0.5)\}
$$

The reliability/survival function

$$
\begin{aligned}
& \mathrm{R}(\mathrm{x} ; \mu, \sigma)=1-\exp \{-\exp (-(\mathrm{x}-\mu) / \sigma)\} ; \\
& \text { where }(\mu, \sigma)>0, \mathrm{x}>0
\end{aligned}
$$
by

The random deviate can be generated from $\operatorname{Gumbel}(\square \mu, \sigma)$

$$
\mathrm{x}=\mu-\sigma[\log (-\log (\mathrm{u}))] \quad ; 0<\mathrm{u}<1 .
$$

Where $\mathrm{u}$ is uniform distribution over $(0,1)$. The associated $\mathrm{R}$ functions for above statistical properties of Gumbel model i.e. pgumbel( ), dgumbel( ), hgumbel( ), qgumbel( ), sgumbel( ) and rgumbel( ) given in [7] can be used for the computation of cdf, pdf, hazard, quantile, reliability and random deviate generation functions respectively. Matrix

Maximum Likelihood Estimation(MLE) and Information

To obtain maximum likelihood estimators of the parameters $(\square \mu, \sigma)$. Let $x 1, \ldots$, xn be a sample from a distribution with cumulative distribution function (2.1). The likelihood function is given by

$$
\log L=-n \log \sigma-\sum_{i=1}^{n}\left(\frac{x_{i}-\mu}{\sigma}\right)-\sum_{i=1}^{n} \exp \left\{-\left(\frac{x_{i}-\mu}{\sigma}\right)\right\}
$$

Therefore, to obtain the MLE's of $\mu$ and $\sigma$ we can maximize directly with respect to $\mu$ and $\sigma$ or we can solve the following two non-linear equations using iterative procedure $[8,9,10$ and 11]:

$$
\begin{aligned}
& \frac{\partial \log \mathrm{L}}{\partial \mu}=\frac{\mathrm{n}}{\sigma}-\frac{1}{\sigma} \sum_{\mathrm{i}=1}^{\mathrm{n}} \exp \left\{-\left(\frac{\mathrm{x}_{\mathrm{i}}-\mu}{\sigma}\right)\right\}=0 \\
& \frac{\partial \log \mathrm{L}}{\partial \sigma}=-\frac{\mathrm{n}}{\sigma}+\sum_{\mathrm{i}=1}^{\mathrm{n}}\left(\frac{\mathrm{x}_{\mathrm{i}}-\mu}{\sigma^{2}}\right)\left[1-\exp \left\{-\left(\frac{\mathrm{x}_{\mathrm{i}}-\mu}{\sigma}\right)\right\}\right]=0
\end{aligned}
$$

\section{A. Asymptotic Confidence bounds. based on MLE}

Since the MLEs of the unknown parameters $\theta \square \square \mu \square \square \square \sigma$ ) cannot be obtained in closed forms, it is not easy to derive the exact distributions of the MLEs. We can derive the asymptotic confidence intervals of these parameters when $\mu$ $\square \square \square \square \square$ and $\square \sigma \square \square \square \square$. The simplest large sample approach is to assume that the $\operatorname{MLE}(\hat{\mu}, \hat{\sigma})$ are approximately bivariate normal with mean $(\mu, \sigma)$ and covariance matrix $\mathrm{I}_{0}^{-1}$, [Lawless(2003)], where $\mathrm{I}_{0}^{-1}$ is the inverse of the observed information matrix

$$
\begin{aligned}
& \mathrm{I}_{0}^{-1}=\left(\begin{array}{cc}
-\left.\frac{\partial^{2} \ln \mathrm{L}}{\partial \mu^{2}}\right|_{\hat{\mu}, \hat{\sigma}}-\left.\frac{\partial^{2} \ln \mathrm{L}}{\partial \mu \partial \sigma}\right|_{\hat{\mu}, \hat{\sigma}}=\left(-\left.\mathrm{H}\right|_{(\hat{\mu}, \hat{\sigma})}\right)^{-1} \\
-\left.\frac{\partial^{2} \ln \mathrm{L}}{\partial \mu \partial \sigma}\right|_{\hat{\mu}, \hat{\sigma}}-\left.\frac{\partial^{2} \ln \mathrm{L}}{\partial \sigma^{2}}\right|_{\hat{\mu}, \hat{\sigma}}
\end{array}\right)^{-1} \\
& =\left(\begin{array}{cc}
\operatorname{var}(\hat{\mu}) & \operatorname{cov}(\hat{\mu}, \hat{\sigma}) \\
\operatorname{cov}(\hat{\mu}, \hat{\sigma}) & \operatorname{var}(\hat{\sigma})
\end{array}\right) .
\end{aligned}
$$


The above approach is used to derive the 100(1 - $\square \square / 2) \%$ confidence intervals of the parameters $\square=(\mu, \sigma)$ as in the following forms

$$
\hat{\mu} \pm z_{\gamma / 2} \sqrt{\operatorname{Var(\hat {\mu })}} \text { and } \hat{\sigma} \pm z_{\gamma / 2} \sqrt{\operatorname{Var(\hat {\sigma })}}
$$

Here, $\mathbf{z} \square / 2$ is the upper ( $\square / 2$ )th percentile of the standard normal distribution.

\section{B. Data Analysis}

In this section we present the analysis of one real data set for illustration of the proposed methodology. The data set contains 36 months of defect-discovery times for a release of Controller Software consisting of about 500,000 lines of code installed on over 100,000 controllers. The defects are those that were present in the code of the particular release of the software, and were discovered as a result of failures reported by users of that release, or possibly of the follow-on release of the product.[13] First we compute the maximum likelihood estimates.

\section{Computation of MLE and model validation}

The Gumbel model is used to fit this data set. We have started the iterative procedure by maximizing the loglikelihood function given in (3.1) directly with an initial guess for $\mu=202.0$ and $\sigma=145.0$, far away from the solution. We have used optim( ) function in $\mathrm{R}$ with option Newton-Raphson method. The iterative process stopped only after 1211 iterations. We obtain $\hat{\mu}=212.1565, \hat{\sigma}=151.7684$ and the corresponding log-likelihood value $=-734.5823$. The similar results are obtained using maxLik package available in $\mathrm{R}$. An estimate of variance-covariance matrix, using (3.4), is given by

$$
\left(\begin{array}{cc}
\operatorname{var}(\hat{\mu}) & \operatorname{cov}(\hat{\mu}, \hat{\sigma}) \\
\operatorname{cov}(\hat{\mu}, \hat{\sigma}) & \operatorname{var}(\hat{\sigma})
\end{array}\right)=\left[\begin{array}{rr}
230.6859 & 53.2964 \\
53.2964 & 133.6387
\end{array}\right]
$$

Thus using (3.5), we can construct the approximate $95 \%$ confidence intervals for the parameters of Gumbel model based on MLE's. Table 1 shows the MLE's with their standard errors and approximate $95 \%$ confidence intervals for $\mu$ and $\sigma$.

TABLE I. MAXIMUM LIKELIHOOD ESTIMATELE(MLE), STANDARD ERROR AND 95\% CONFIDENCE INTERVAL

\begin{tabular}{|c|c|c|c|}
\hline Parameter & MLE & Std. Error & 95\% Confidence Interval \\
\hline $\boldsymbol{m u}$ & 212.1565 & 15.188 & $(182.38802,241.92498)$ \\
\hline sigma & 151.7684 & 11.560 & $(93.1108,174.426)$ \\
\hline
\end{tabular}

To check the validity of the model, we compute the Kolmogorov-Smirnov (KS) distance between the empirical distribution function and the fitted distribution function when the parameters are obtained by method of maximum likelihood. For this we can use R function ks.gumbel( ), given in [7]. The result of K-S test is $\mathrm{D}=0.0699$ with the corresponding p-value $=0.0 .6501$, therefore, the high $\mathrm{p}$-value clearly indicates that Gumbel model can be used to analyze this data set. We also plot the empirical distribution function and the fitted distribution function in Fig. 2.

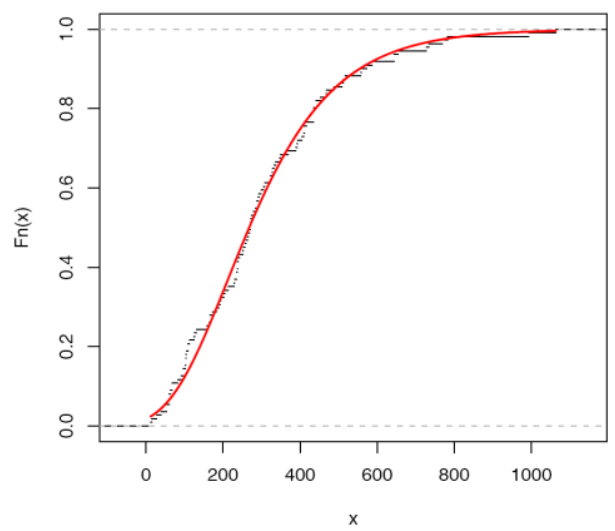

Figure 2. The graph of empirical distribution function and fitted distribution function.

Therefore, it is clear that the estimated Gumbel model provides excellent good fit to the given data.

\section{Bayesian Estimation in OpenBUGS}

A module dgumbel(mu, sigma) is written in component Pascal, given in [13] enables to perform full Bayesian analysis of Gumbel model into OpenBUGS using the method described in $[14,15]$.

\section{1) Bayesian Analysis under Uniform Priors}

The developed module is implemented to obtain the Bayes estimates of the Gumbel model using MCMC method. The main function of the module is to generate MCMC sample from posterior distribution for given set of uniform priors. Which is frequently happens that the experimenter knows in advance that the probable values of $\square$ lie over a finite range [a, b] but has no strong opinion about any subset of values over this range. In such a case a uniform distribution over $[a, b]$ may be a good approximation of the prior distribution, its p.d.f. is given by

$$
\pi(\theta)= \begin{cases}\frac{1}{\mathbf{b}-\mathbf{a}} & ; 0<\mathbf{a} \leq \mathbf{\theta} \leq \mathbf{b} \\ \mathbf{0} & ; \text { otherwise }\end{cases}
$$

We run the two parallel chains for sufficiently large number of iterations, say 5000 the burn-in, until convergence results. Final posterior sample of size 7000 is taken by choosing thinning interval five i.e. every fifth outcome is stored.

Therefore, we have the posterior sample $\{\alpha 1 \mathrm{i}, \lambda 1 \mathrm{i}\}, \mathrm{i}=$ $1, \ldots, 7000$ from chain 1 and $\{\alpha 2 \mathrm{i}, \lambda 2 \mathrm{i}\}, \mathrm{i}=1, \ldots, 7000$ from chain 2 .

The chain 1 is considered for convergence diagnostics plots. The visual summary is based on posterior sample obtained from chain 2 whereas the numerical summary is presented for both the chains.

\section{E. Convergence diagnostics}

Before examining the parameter estimates or performing other inference, it is a good idea to look at plots of the sequential(dependent) realizations of the parameter estimates and plots thereof. We have found that if the Markov chain is not mixing well or is not sampling from the stationary 
distribution, this is usually apparent in sequential plots of one or more realizations. The sequential plot of parameters is the plot that most often exhibits difficulties in the Markov chain.

- $\quad$ History(Trace) plot
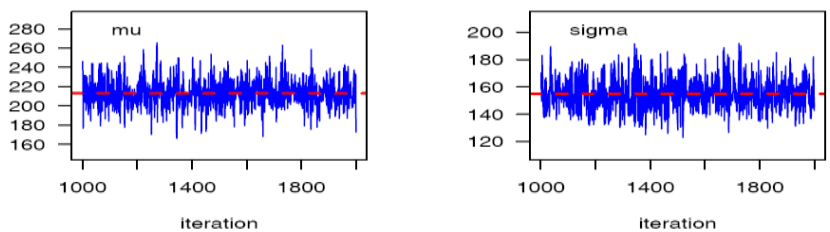

Figure 3. Sequential realization of the parameters $\mu$ and $\sigma$.

Fig. 3 shows the sequential realizations of the parameters of the model. In this case Markov chain seems to be mixing well enough and is likely to be sampling from the stationary distribution. The plot looks like a horizontal band, with no long upward or downward trends, then we have evidence that the chain has converged.

- $\quad$ Running Mean (Ergodic mean) Plot

In order to study the convergence pattern, we have plotted a time series (iteration number) graph of the running mean for each parameter in the chain. The mean of all sampled values up to and including that at a given iteration gives the running mean. In the Fig. 4 given below, a systematic pattern of convergence based on ergodic averages can be seen after an initial transient behavior of the chain.
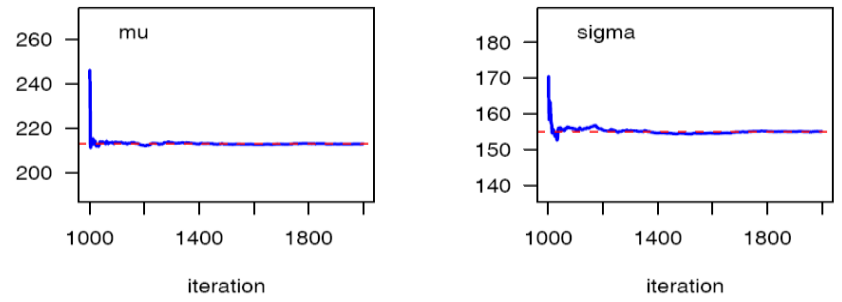

Figure 4. The Ergodic mean plots for mu and sigma.

\section{F. Numerical Summary}

TABLE 2: NUMERICAL SUMMARIES BASED ON MCMC SAMPLE OF POSTERIOR CHARACTERISTICS FOR GUMBEL MODEL UNDE

\begin{tabular}{|l|c|c|c|c|}
\hline \multirow{2}{*}{ Characteristics } & \multicolumn{2}{|c}{ Chain 1 } & \multicolumn{2}{c|}{ Chain 2 } \\
\cline { 2 - 5 } & mu & sigma & mu & sigma \\
\hline Mean & 212.7371 & 154.524 & 212.8164 & 154.8901 \\
\hline Standard Deviation & 15.42162 & 11.91967 & 15.60924 & 11.78936 \\
\hline Monte Carlo(MC) error & 0.1899 & 0.1341 & 0.1913 & 0.106 \\
\hline Minimum & 156.20 & 120.2 & 153.9 & 115.9 \\
\hline 2.5th Percentile $\left(\mathrm{P}_{2.5}\right)$ & 181.90 & 133.0 & 182.4 & 133.6 \\
\hline First Quartile $\left(\mathrm{Q}_{1}\right)$ & 202.60 & 146.3 & 202.2 & 146.8 \\
\hline Median & 212.75 & 153.8 & 212.5 & 154.3 \\
\hline Third Quartile $\left(\mathrm{Q}_{3}\right)$ & 222.80 & 162.4 & 223.0 & 162.5 \\
\hline 97.5 ${ }^{\text {th }}$ Percentile $\left(\mathrm{P}_{97.5}\right)$ & 242.80 & 179.1 & 244.3 & 179.7 \\
\hline Maximum & 275.20 & 209.3 & 279.9 & 203.0 \\
\hline Mode & 212.7101 & 150.7500 & 210.9984 & 155.1407 \\
\hline 95\% Credible Interval & $(181.9,242.8)$ & $(133.0,179.1)$ & $(182.4,244.3)$ & $(133.6,179.7)$ \\
\hline 95\% HPD Crd Interval & $(181.0,241.5)$ & $(132.9,178.9)$ & $(180.8,242.6)$ & $(133.2,179.1)$ \\
\hline
\end{tabular}

In Table 2, we have considered various quantities of interest and their numerical values based on MCMC sample of posterior characteristics for Gumbel model under uniform priors. The numerical summary is based on final posterior sample (MCMC output) of 7000 samples for mu and sigma.

$$
\begin{aligned}
& \left\{\mu_{1 \mathrm{i}}, \sigma_{1 \mathrm{i}}\right\}, \quad \mathrm{i}=1, \ldots, 7000 \text { from chain } 1 \text { and } \\
& \left\{\mu_{2 \mathrm{i}}, \square \sigma_{2 \mathrm{i}}\right\}, \mathrm{i}=1, \ldots, 7000 \text { from chain } 2 .
\end{aligned}
$$

\section{G. Visual summary by using Box plots}

The boxes represent in Fig. 5, inter-quartile ranges and the solid black line at the (approximate) centre of each box is the mean; the arms of each box extend to cover the central 95 per cent of the distribution - their ends correspond, therefore, to the $2.5 \%$ and $97.5 \%$ quantiles. (Note that this representation differs somewhat from the traditional.
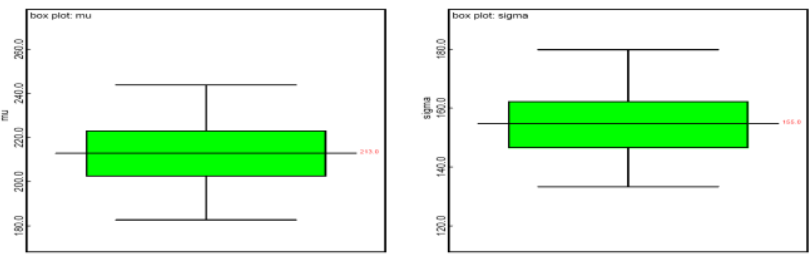

Figure 5. The boxplots for mu and sigma

\section{2) Bayesian Analysis under Gamma Priors}

The developed module is implemented to obtain the Bayes estimates of the Gumbel model using MCMC method to generate MCMC sample from posterior distribution for given set of gamma priors, which is most widely used prior distribution of $\theta$ is the inverted gamma distribution with parameters $a$ and $b(>0)$ with p.d.f. given by

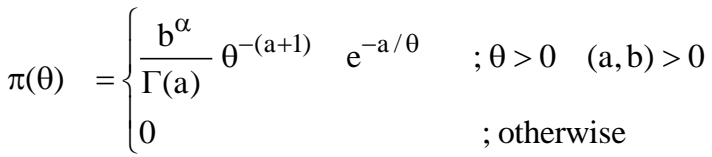

We also run the two parallel chains for sufficiently large number of iterations, say 5000 the burn-in, until convergence results. Final posterior sample of size 7000 is taken by choosing thinning interval five i.e. every fifth outcome is stored and same procedure is adopted for analysis as used in the case of uniform priors.

\section{H. Convergence diagnostics}

Simulation-based Bayesian inference requires using simulated draws to summarize the posterior distribution or calculate any relevant quantities of interest. We need to treat the simulation draws with care. Trace plots of samples versus the simulation index can be very useful in assessing convergence. The trace indicates if the chain has not yet converged to its stationary distribution - that is, if it needs a longer burn-in period. A trace can also tell whether the chain is mixing well. A chain might have reached stationary if the distribution of points is not changing as the chain progresses. The aspects of stationary that are most recognizable from a trace plot are a relatively constant mean and variance.

\section{- Autocorrelation}

The graph shows that the correlation is almost negligible. We may conclude that the samples are independent. 

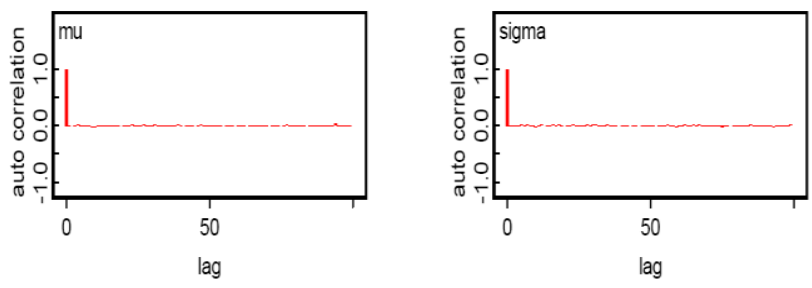

Figure 6. The autocorrelation plots for mu and sigma.

\section{- Brooks-Gelman-Rubin}

Uses parallel chains with dispersed initial values to test whether they all converge to the same target distribution. Failure could indicate the presence of a multi-mode posterior distribution (different chains converge to different local modes) or the need to run a longer chain (burn-in is yet to be completed).
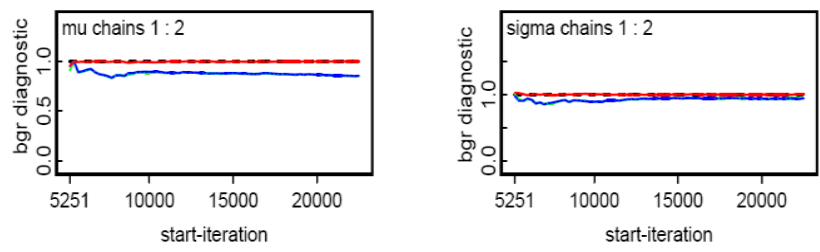

Figure 7. The BGR plots for mu and sigma

From the Fig. 7, it is clear that convergence is achieved. Thus we can obtain the posterior summary statistics.

\section{NUMERICAL SUMMARY}

In Table 3, we have considered various quantities of interest and their numerical values based on MCMC sample of posterior characteristics for Gumbel model under Gamma priors.

\begin{tabular}{|c|c|c|c|c|}
\hline \multirow{2}{*}{ Characteristics } & \multicolumn{2}{|c|}{ Chain 1} & \multicolumn{2}{|c|}{ Chain 2} \\
\hline & $\mathrm{mu}$ & sigma & $\mathrm{mu}$ & sigma \\
\hline Mean & 211.0273 & 153.4008 & 211.2025 & 153.1235 \\
\hline Standard Deviation & 15.53698 & 11.77100 & 15.18645 & 11.64925 \\
\hline Monte Carlo(MC) error & 0.1729 & 0.1475 & 0.1831 & 0.1233 \\
\hline Minimum & 153.0 & 117.5 & 156.4 & 113.7 \\
\hline 2.5th Percentile $\left(\mathrm{P}_{2.5}\right)$ & 180.6 & 131.2 & 182.0 & 131.9 \\
\hline First Quartile $\left(\mathrm{Q}_{1}\right)$ & 200.7 & 145.4 & 200.8 & 145.1 \\
\hline Median & 210.9 & 152.9 & 211.2 & 152.6 \\
\hline Third Quartile $\left(\mathrm{Q}_{3}\right)$ & 221.4 & 160.9 & 221.4 & 160.5 \\
\hline 97.5th Percentile $\left(\mathrm{P}_{97.5}\right)$ & 242.1 & 178.0 & 241.2 & 177.6 \\
\hline Maximum & 267.5 & 200.2 & 268.3 & 210.4 \\
\hline Mode & 209.8722 & 151.0876 & 209.7639 & 152.919 \\
\hline $95 \%$ Credible Interval & $(180.6,242.1)$ & $(131.2,178.0)$ & $(182.0,241.2)$ & $(131.9,177.6)$ \\
\hline $95 \%$ HPD Credible Interval & $(180.5,241.8)$ & $(129.0,175.7)$ & $(181.5,240.5)$ & $(130.9,176.4)$ \\
\hline
\end{tabular}

\section{A. Visual summary by using Kernel density estimates}

Histograms can provide insights on skewness, behaviour in the tails, presence of multi-modal behaviour, and data outliers; histograms can be compared to the fundamental shapes associated with standard analytic distributions.

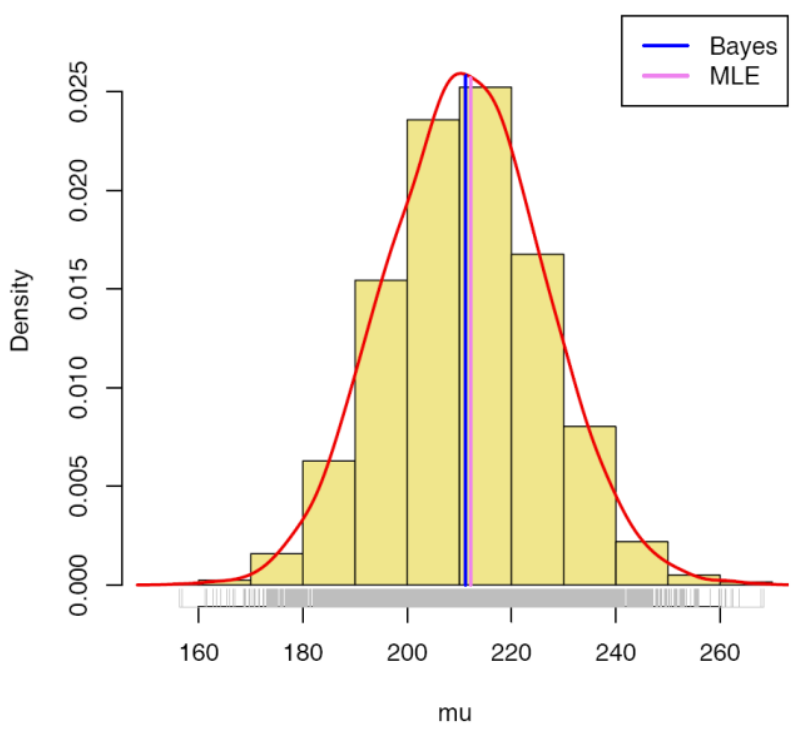

Figure 8. Histogram and kernel density estimate of $\mu$ based on MCMC samples, vertical lines represent the corresponding MLE and Bayes estimate.

Fig. 8 and Fig. 9 provide the kernel density estimate of $\mu$ and $\sigma$. The kernel density estimates have been drawn using $R$ with the assumption of Gaussian kernel and properly chosen values of the bandwidths. It can be seen that $\mu$ and $\sigma$ both are symmetric.

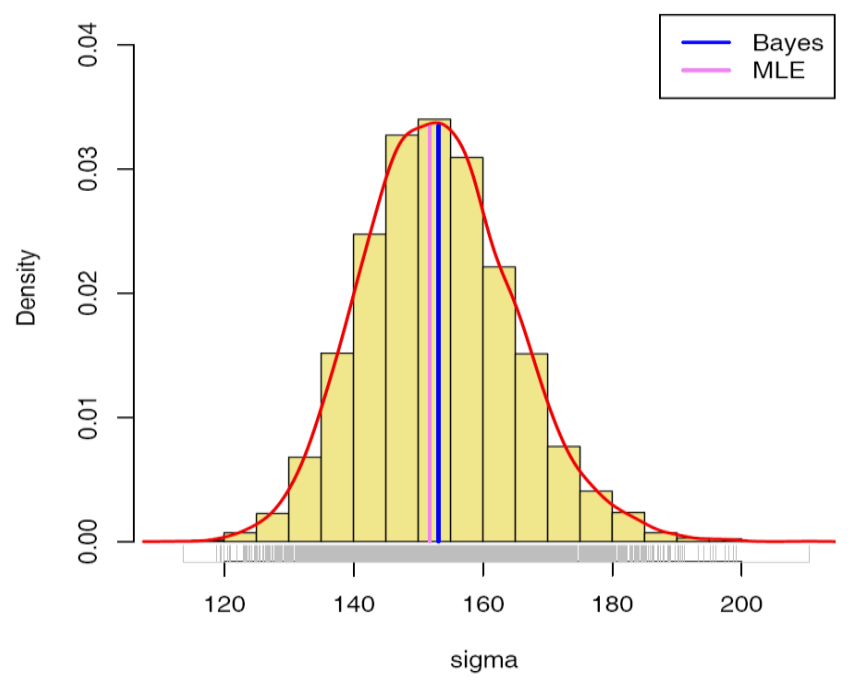

Figure 9. Histogram and kernel density estimate of $\sigma$ based on MCMC samples, vertical lines represent the corresponding MLE and Bayes estimate.

\section{B. Comparison with MLE using Uniform Priors}

For the comparison with MLE we have plotted two graphs. In Fig. 10, the density functions $\mathrm{f}(\mathrm{x} ; \hat{\mu}, \hat{\sigma})$ using MLEs and Bayesian estimates, computed via MCMC samples under uniform priors, are plotted. 


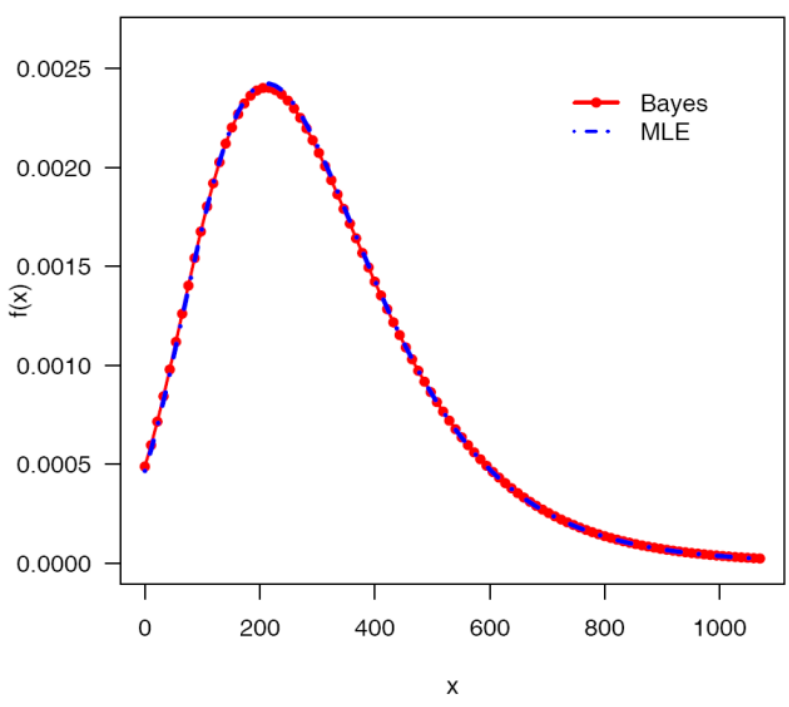

Figure 10. The density functions $f(x ; \hat{\mu}, \hat{\sigma})$ using MLEs and Bayesian estimates, computed via MCMC samples.

Whereas, Fig.11 represents the Quantile-Quantile(Q-Q) plot of empirical quantiles and theoretical quantiles computed from MLE and Bayes estimates.

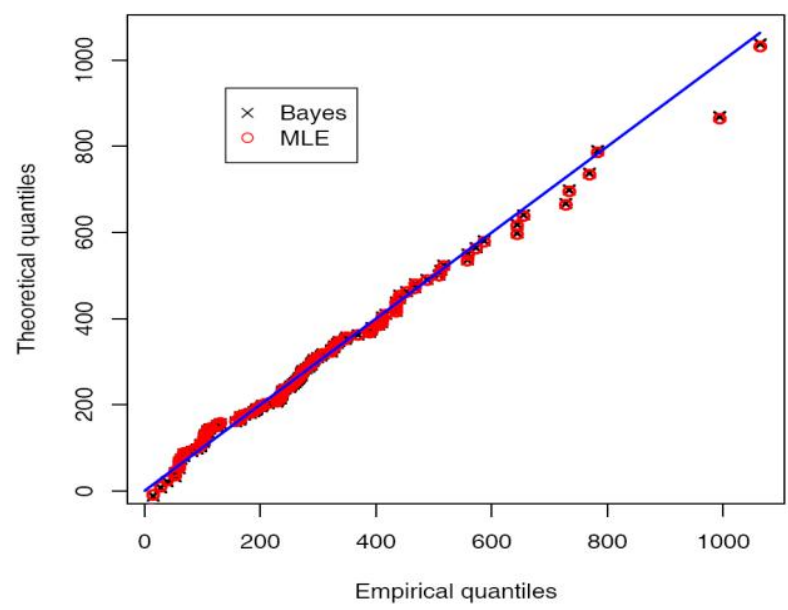

Figure 11. Quantile-Quantile(Q-Q) plot of empirical quantiles and theoretical quantiles computed from MLE and Bayes estimates.

It is clear from the Figures, the MLEs and the Bayes estimates with respect to the uniform priors are quite close and fit the data very well.

\section{Comparison with MLE using Gamma Priors}

For the comparison with MLE, we have plotted a graph which exhibits the estimated reliability function (dashed line) using Bayes estimate under gamma priors and the empirical reliability function(solid line). It is clear from Fig.12, the MLEs and the Bayes estimates with respect to the gamma priors are quite close and fit the data very well.

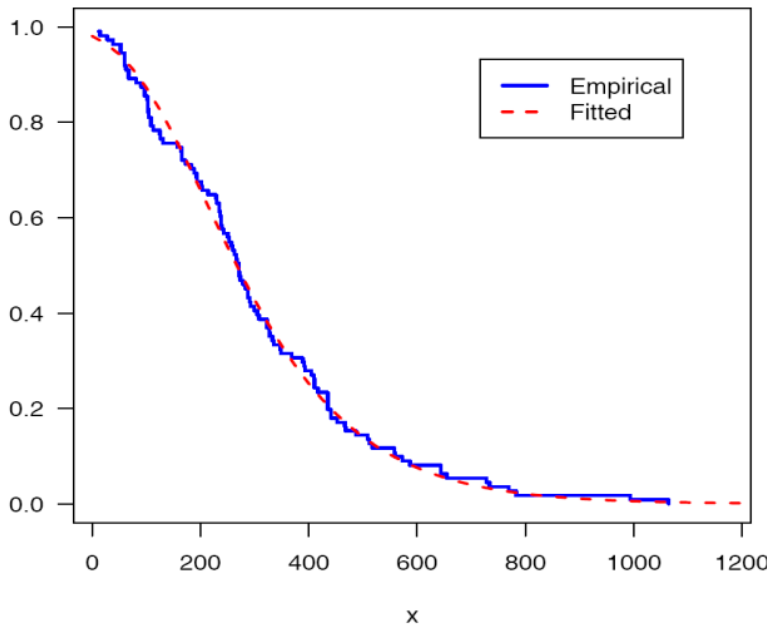

Figure 12. The estimated reliability function(dashed line) and the empirical reliability function (solid line).

\section{CONCLUSION}

The developed methodology for MLE and Bayesian estimation has been demonstrated on a real data set when both the parameters mu (location) and sigma (scale) of the Gumbel model are unknown under non-informative and informative set of independent priors. The bayes estimates of the said priors, i.e., uniform and gamma have been obtained under squared error, absolute error and zero-one loss functions. A five point summary Minimum ( $\mathrm{x}$ ), $\mathrm{Q}_{1}, \mathrm{Q}_{2}, \mathrm{Q}_{3}$, Maximum (x) has been computed. The symmetric Bayesian credible intervals and Highest Probability Density (HPD) intervals have been constructed. Through the use of graphical representations the intent is that one can gain a perspective of various meanings and associated interpretations.

The MCMC method provides an alternative method for parameter estimation of the Gumbel model. It is more flexible when compared with the traditional methods such as MLE method. Moreover, 'exact' probability intervals are available rather than relying on estimates of the asymptotic variances. Indeed, the MCMC sample may be used to completely summarize posterior distribution about the parameters, through a kernel estimate. This is also true for any function of the parameters such as hazard function, mean time to failure etc. The MCMC procedure can easily be applied to complex Bayesian modeling relating to Gumbel model

\section{ACKNOWLEDGMENT}

The authors are thankful to the editor and the referees for their valuable suggestions, which improved the paper to a great extent.

\section{REFERENCES}

[1] Gumbel, E.J.(1954). Statistical theory of extreme values and some practical applications. Applied Mathematics Series, 33. U.S. Department of Commerce, National Bureau of Standards. 
[2] Coles, Stuart (2001). An Introduction to Statistical Modeling of Extreme Values,. Springer-Verlag. ISBN 1-85233-459-2.

[3] Wu, J.W., Hung, W.L., Tsai, C.H.(2004). Estimation of parameters of the Gompertz distribution using the least squares method, Applied Mathematics and Computation 158 (2004) 133-147

[4] Cid, J. E. R. and Achcar, J. A., (1999). Bayesian inference for nonhomogeneousPoisson processes in software reliability models assuming nonmonotonic intensityfunctions, Computational Statistics and Data Analysis, 32, 147-159.

[5] Murthy, D.N.P., Xie, M., Jiang, R. (2004). Weibull Models, Wiley, New Jersey.

[6] Srivastava, A.K. and Kumar V. (2011). Analysis of software reliability data usingexponential power model. International Journal of Advanced Computer Science and Applications, Vol. 2(2), 38-45.

[7] Kumar, V. and Ligges, U. (2011). reliaR : A package for some probability distributions. http://cran.r-project.org/web/packages/reliaR/ index.html.

[8] Chen, Z., A new two-parameter lifetime distribution with bathtub shape or increasing failure rate function, Statistics \& Probability Letters, Vol.49, pp.155-161, 2000.

[9] Wang, F. K., A new model with bathtub-shaped failure rate using an additive Burr XII distribution, Reliability Engineering and System Safety, Vol.70, pp.305-312, 2000.

[10] Srivastava, A.K. and Kumar V. (2011). Markov Chain Monte Carlo methods for Bayesian inference of the Chen model, International Journal of Computer Information Systems, Vol. 2 (2), 07-14.

[11] Srivastava, A.K. and Kumar V. (2011). Software reliability data analysis with Marshall-Olkin Extended Weibull model using MCMC method for non-informative.

[12] Lawless, J. F., (2003). Statistical Models and Methods for Lifetime Data, 2nd ed., John Wiley and Sons, New York.

[13] Lyu, M.R., (1996). Handbook of Software Reliability Engineering, IEEE Computer Society Press, McGraw Hill, 1996.

[14] Kumar, V., Ligges, U. and Thomas, A. (2010). ReliaBUGS User Manual : A subsystem in OpenBUGS for some statistical models, Version 1.0, OpenBUGS 3.2.1, http://openbugs.info/w/Downloads/.
[15] Thomas, A. (2010). OpenBUGS Developer Manual, Version 3.1.2, http://www.openbugs.info/.

[16] Chen, M., Shao, Q. and Ibrahim, J.G. (2000). Monte Carlo Methods in Bayesian Computation, Springer, NewYork.

\section{AUTHORS PROFILE}

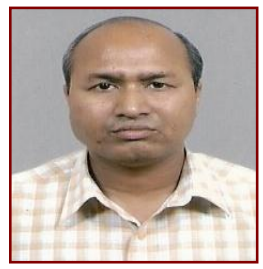

RAJ KUMAR received his MCA from M.M.M Engineering College, Gorakhpur and perusing Ph.D. in Computer Science from D. D.U. Gorakhpur University. Currently working in National Institute of Electronics and Information Technology (formly known as DOEACC Society), Gorakhpur, Ministry of Communication and Information Technology, Government of India.

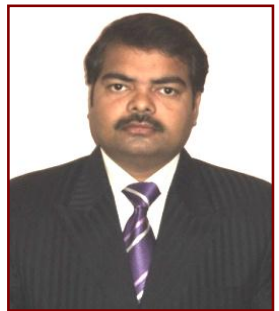

ASHWINI KUMAR SRIVASTAVA received his M.Sc in Mathematics from D.D.U.Gorakhpur University, MCA(Hons.) from U.P.Technical University, M. Phil in Computer Science from Allagappa University and Ph.D. in Computer Science from D.D.U.Gorakhpur University, Gorakhpur. Currently working as Assistant Professor in Department of Computer Application in Shivharsh Kisan P.G. College, Basti, U.P. He has got 8 years of teaching experience as well as 4 years research experience. His main research interests are Software Reliability, Artificial Neural Networks, Bayesian methodology and Data Warehousing.

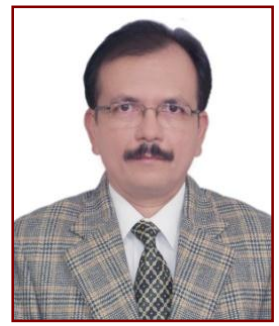

VIJAY KUMAR received his M.Sc and Ph.D. in Statistics from D.D.U. Gorakhpur University. Currently working as Associate Professor in Department of Maths. and Statistics in DDU Gorakhpur University, Gorakhpur. He has got 17 years of teaching/research experience. He is visiting Faculty of Max-Planck-Institute, Germany. His main research interests are Bayesian statistics, reliability models and computational statistics using OpenBUGS and R. 